\title{
Fatigue crack initiation in nickel-based superalloys studied by microstructure-based FE modeling and scanning electron microscopy
}

\author{
M. Fried ${ }^{1, \text { a }}$, C. Krechel ${ }^{2,3}$, E.E. Affeldt ${ }^{1}$, B. Eckert ${ }^{1,4}$, S. Kimmig ${ }^{1}$, U. Retze ${ }^{1}$, H.W. Höppel ${ }^{2,3}$, and M. Göken ${ }^{2,3}$ \\ ${ }^{1}$ MTU Aero Engines AG, 80995 Munich, Germany \\ ${ }^{2}$ Friedrich-Alexander-Universität Erlangen-Nürnberg, Department of Materials Science and Engineering, Institute of \\ General Material Properties WW1, 91058 Erlangen, Germany \\ ${ }^{3}$ Zentralinstitut für Neue Materialien und Prozesstechnik ZMP, 90762 Fürth, Germany \\ ${ }^{4}$ Now at University of Applied Sciences Augsburg, Faculty of Electrical Engineering, 86161 Augsburg, Germany
}

\begin{abstract}
In this work stage I crack initiation in polycrystalline nickel-based superalloys is investigated by analyzing anisotropic mechanical properties, local stress concentrations and plastic deformation on the microstructural length scale. The grain structure in the gauge section of fatigue specimens was characterized by EBSD. Based on the measured data, a microstructure-based FE model could be established to simulate the strain and stress distribution in the specimens during the first loading cycle of a fatigue test. The results were in fairly good agreement with experimentally measured local strains. Furthermore, the onset of plastic deformation was predicted by identifying shear stress maxima in the microstructure, presumably leading to activation of slip systems. Measurement of plastic deformation and observation of slip traces in the respective regions of the microstructure confirmed the predicted slip activity. The close relation between micro-plasticity, formation of slip traces and stage I crack initiation was demonstrated by SEM surface analyses of fatigued specimens and an in-situ fatigue test in a large chamber SEM.
\end{abstract}

\section{Introduction}

Due to their outstanding strength and thermal stability at high temperatures, nickel-based superalloys are commonly used as airfoil materials in aero engine turbines [1]. Since these components undergo variable thermal and mechanical loads during service, a sound assessment of the material's behavior under cyclic loading is required.

For a closer look at fatigue life it is convenient to distinguish two phenomena: crack initiation and crack propagation. The total life to failure may be decomposed into a crack initiation and a crack propagation phase [2]. Miller et al. have shown that with decreasing load level the initiation phase becomes dominant [3]. This behavior can be regarded to be typical for high cycle fatigue (HCF) conditions, where the imposed deformation mostly generate a macroscopically elastic material response.

Due to the important role of crack initiation in the HCF regime, a close look to the relevant damage mechanisms is required. Two kinds of material characteristics can be distinguished: Weidner et al. [4] have described crack initiation in a material almost free of heterogeneities. It was shown that irreversible dislocation motion induced formation of persistent slip bands, which lead to roughening of the specimen surface and thus to formation of stage I cracks. Consequently, crack formation was attributed to localized micro-plastic deformation. On the

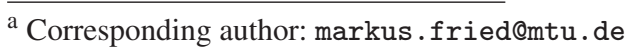

other hand, Murakami et al. [5] have described crack initiation starting from inclusions, growing to microcracks until critical stress concentrations induced crack propagation. A large portion of the total specimen life at low load levels is consumed by the crack initiation period.

At higher loads approaching the macroscopic yield point of the material, the life to failure is typically governed by crack propagation, as for low cycle fatigue (LCF). Many authors, e.g. Neumann et al. [6] and Seifert et al. [7] have shown, that life to failure can be modeled based on the cyclic deformation data. In the model the initiating crack size represents an essential parameter.

It must be pointed out that the damage mechanisms observed under LCF or HCF conditions largely depend on the microstructure of the material. In the case of nickelbased superalloys inherent heterogeneities such as pores and inclusions (e.g. carbides) play an important role as potential crack initiation sites. However, also stage I cracks initiating from slip bands are found. Thus, only a close look to the material's microstructure in combination with a description of local plasticity on the length scale of the microstructural features provides an adequate basis for a better understanding of crack initiation.

As an approach to predict fatigue crack initiation sites in bi- and polycrystals, Vehoff et al. [8] proposed an anisotropic elastic model to calculate stress concentrations due to grain stiffness incompatibility. Their experiments on Fe-Si alloys showed that cracks initiated preferably at

This is an Open Access article distributed under the terms of the Creative Commons Attribution License 4.0, which permits unrestricted use, distribution, and reproduction in any medium, provided the original work is properly cited. 


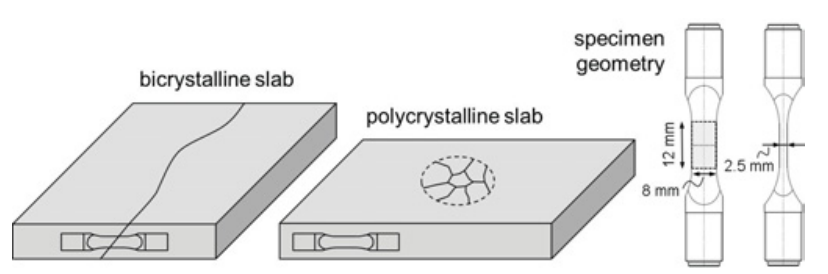

Figure 1. Material and specimens used for this investigation.
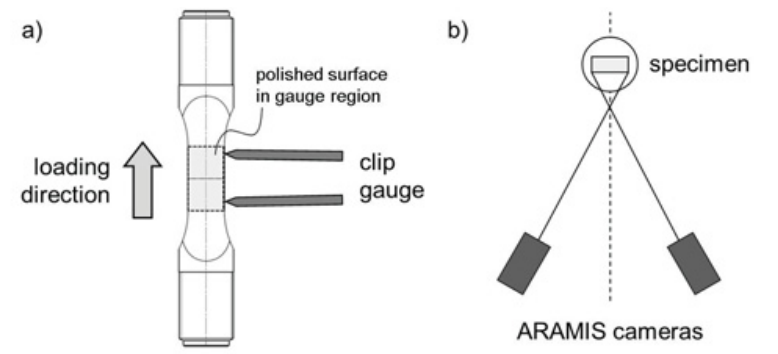

Figure 2. Fatigue test setup, a) front view, b) top view.

grain boundaries coinciding with simulated local stress concentrations. In another work by Oja et al. [9] it is proposed that additionally to elastic incompatibility, also anisotropic plastic properties can be an important factor for crack initiation in nickel-based polycrystals. Their experiments indicated that crack initiation occurred either in large grains with high Schmid factors or within groups of grains with similar orientations.

In this paper, a microstructure-based analysis will be presented considering the elastic and plastic anisotropic material behavior. The capability of this model to predict the local onset of plastic deformation in nickel-based superalloys will be demonstrated by comparing simulation results to experiments. The relevance of micro-plasticity for stage I crack initiation will be discussed.

\section{Experimental procedure}

\subsection{Materials used in this study}

For the experiments on polycrystalline samples the commercial nickel-based superalloy Inconel ${ }^{\circledR} 100$ was cast to slabs of $\sim 300 \mathrm{~mm} \times 150 \mathrm{~mm} \times 20 \mathrm{~mm}$. Bicrystalline specimens were produced from a slab of M-247LC SX, an alloy developed for single crystalline castings. The slab was grown from two crystal seeds of different orientation, so a single high angle grain boundary formed during solidification in the middle of the slab. From the cast slabs specimen blanks were cut and machined to final shape by turning and electrical discharge machining.

\subsection{Grain orientation measurement}

The specimen surface in the gauge section (Fig. 2a) was first mechanically and then electrolytically polished. The measurements were carried out with a Tescan Vega XMU scanning electron microscope at a magnification of 100 with a working distance of $25 \mathrm{~mm}$ and a spot size of $830 \mathrm{~nm}$. The grain orientation was measured using an electron backscatter diffraction (EBSD) detector (HKL Channel 5). The area for orientation mapping was set

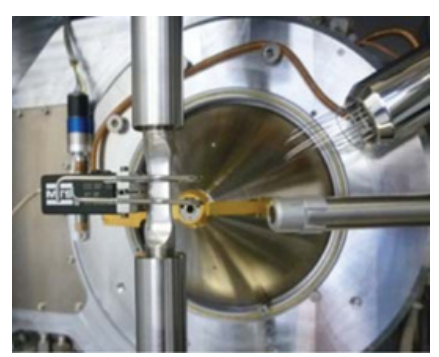

Figure 3. Specimen mounted in the servo-hydraulic test rig of the large chamber SEM at the ZMP Fürth.

to approx. $2800 \mu \mathrm{m} \times 1800 \mu \mathrm{m}$ (step width $40 \mu \mathrm{m}$ ). In order to cover the complete gauge section, the specimen was shifted several times in horizontal and vertical direction. The separate mappings were finally merged to one orientation map using the HKL MapStitcher software.

\subsection{Fatigue tests with local strain measurement}

The fatigue tests were carried out in a servo-hydraulic testing machine in strain-controlled mode using an MTS controller. The macroscopic strain was measured with a $12 \mathrm{~mm}$ clip gauge clamped to one side of the specimen. The tests were performed in air at ambient temperature.

The local strain distribution on the specimen surface was measured using the ARAMIS system from GOM. Two cameras were placed in front of the test setup as sketched in Fig. 2b. A random dot pattern was applied on the specimen surface with a spray technique. Comparing images of the deformed state with an undeformed reference, the local strain distribution was calculated by the GOM software.

\subsection{In-situ fatigue tests in a large chamber SEM}

Further fatigue tests were carried out in the large chamber SEM (LC-SEM) at Zentralinstitut für Neue Materialien und Prozesstechnik ZMP, Friedrich-Alexander-Universität Erlangen-Nürnberg, in Fürth, Germany. The LC-SEM features a large vacuum chamber $\left(\sim 2 \mathrm{~m}^{3}\right)$ and is equipped with detectors for various kinds of specimen analysis. What makes this setup a unique instrument is the integration of a servo-hydraulic testing device, so analyses can be performed under static or cyclic loading of specimens. Thus, the large chamber SEM is a well-suited instrument for in-situ investigation of fatigue damage mechanisms. For details see references [10] and [11].

\section{Analyses}

\subsection{Orientation data}

The data gained from the EBSD mapping were further processed with the software MATLAB ${ }^{\mathrm{R}}$ [12]. The grain structure was reconstructed from the raw data using the toolbox MTEX developed by Bachmann, Hielscher and Schäben [13]. Then, the grain boundary coordinates and grain orientations were extracted and further processed for specific analyses using a self-written code. The grain orientation was stored in form of Euler angles. From these angles a grain orientation matrix $\mathbf{B}$ can be established using the convention of Bunge [14]. By applying B to any 
vector of the specimen coordinate system, its orientation in the local crystal system is obtained.

\subsection{Elastic stiffness}

As a first approach the local stiffness due to individual grain orientations is regarded. When the grain orientation is known, its stiffness can be calculated. For the uniaxial stiffness $E$ in $\langle l m n\rangle$ direction, equation 1 yields

$$
E=\left\{s_{11}-2\left[\left(s_{11}-s_{12}\right)-0.5 s_{44}\right]\left(l^{2} m^{2}+m^{2} n^{2}+l^{2} n^{2}\right)\right\}^{-1},
$$

where $l, m$ and $n$ are the components of a vector $\mathbf{u}$ in the crystal system. $\mathbf{u}$ is obtained by Eq. (2),

$$
\mathbf{u}=\mathbf{B v}
$$

where $\mathbf{v}$ is the loading direction in the global system. $s_{11}$, $s_{12}$ and $s_{44}$ represent the independent constants forming the compliance matrix, taken from measurements on a nickelbased single crystal alloy published by Hermann [15].

\subsection{Onset of plasticity}

Plastic deformation in any crystalline material involves dislocation slip occurring on slip systems. In order to identify the local onset of plasticity in a polycrystal, the resolved shear stresses $\tau^{\alpha, \mathrm{i}}$ on each slip system (indexed by $\alpha$ ) must be regarded for all grains (indexed by $i$ ). Once a critical shear stress $\tau_{c}$ is reached, plastic deformation occurs. For the nickel fcc crystal twelve octahedral slip systems will be regarded, composed of four slip planes, characterized by a slip plane normal $\mathbf{N}^{\alpha, \mathrm{i}}$, each comprising three slip directions $\mathbf{S}^{\alpha, i}$. Equation 3 transforms the stress tensor $\sigma$ defined in the global system into a shear stress for the respective slip system:

$$
\tau^{\alpha, \mathrm{i}}=\sigma \mathbf{N}^{\alpha, \mathrm{i}} \otimes \mathbf{S}^{\alpha, \mathrm{i}},
$$

with $\alpha \in[1 ; 12], i \in[1 ; n]$ and $n$ the number of grains in the microstructure. $\mathbf{N}^{\alpha, i}$ and $\mathbf{S}^{\alpha, i}$ must be unit vectors. A slip system is active if the condition $\tau^{\alpha, i} \geq \tau_{c}$ is fulfilled.

In the specific case that pure uniaxial stress $\sigma$ can be assumed, the resolved shear stress on a slip system $\alpha$ in grain $i$ can be simply calculated using Eq. (4):

$$
\tau^{\alpha, \mathrm{i}}=s^{\alpha, \mathrm{i}} \sigma
$$

where $s^{\alpha, i}$ is the so-called Schmid factor defined as

$$
s^{\alpha, \mathrm{i}}=\left(\mathbf{B L} \cdot \mathbf{N}^{\alpha, \mathrm{i}}\right)\left(\mathbf{B L} \cdot \mathbf{S}^{\alpha, \mathrm{i}}\right),
$$

with $\mathbf{L}$ the uniaxial loading direction. All vectors in Eq. (5) must be unit vectors. The maximum Schmid factor of all slip systems in grain $i$ is designated $s^{\max , \mathrm{i}}$.

When slip occurs, dislocations move through the material. If the Burgers vector has a component normal to the specimen surface, microscopic steps are formed, referred to as slip marks (cf. Blochwitz et al. [16]). In SEM examination those slip marks appear as straight lines, representing the intersection of the active slip plane with the specimen surface. Their orientation angle $\theta^{\alpha, \mathrm{i}}$ relative

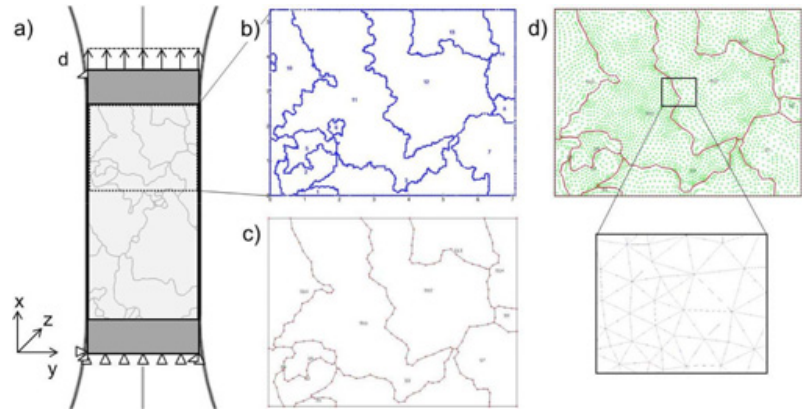

Figure 4. a) FEM boundary conditions, b) original microstructure data, c) geometry prepared for FEM, d) meshed structure.

to the loading direction $\mathbf{L}$ can be calculated using Eq. (6) (cf. Dunne et al. [17]).

$$
\theta^{\alpha, \mathrm{i}}=\operatorname{acos}\left\{\left(\mathbf{B K} \times \mathbf{N}^{\alpha, \mathrm{i}}\right)(\mathbf{B L})\right\},
$$

where $\mathbf{K}$ is the unit vector describing the surface normal in the specimen coordinate system.

\section{Modeling}

In order to simulate the elastic stress and strain distribution, a microstructure-based FE model was established. For that purpose the microstructure measured by EBSD was converted to a 2-dimensional model including both the grain boundary geometry and the grain orientation.

Each grain was modelled as a polygon $s_{\mathrm{k}}$ formed by lines $l_{\mathrm{k}}$ connecting a number of points $p_{\mathrm{k}}$ on the grain boundaries. The coordinates of $p_{\mathrm{k}}$ were extracted from the MTEX grain reconstruction. Since the coordinates essentially follow the measurement grid of the EBSD mapping, the points have a distance close to the beam step width (cf. Sect. 2.2). This would create high mesh density and potentially inhomogeneous node distribution in the FEM mesh. Therefore, the geometrical parameters of the model had to be modified in order to gain better control on the mesh homogeneity. In this work an algorithm was developed to reduce the number of $p_{\mathrm{k}}$, i.e. the grain boundaries were approximated by straight lines. In a further step, a smoothening algorithm was created to prevent sharp edges forming at specific points $p_{\mathrm{k}}$, since this may lead to artificial stress concentrations.

The modeling algorithm also offers the possibility to remove selected grains, with the intention to reduce complexity of the model. The area of the deleted grain is merged with one of its neighbors and its orientation information is neglected. This option was only applied when models incorporating those grains revealed low influence on the mechanical behavior. The step from the original data to a an FE mesh is illustrated in Figs. 4b and $\mathrm{c}$.

The FEM mesh was created using the mesh2D algorithm [18] implemented to CalculiX FEM preprocessing tool [19]. An unstructured mesh of six-node quadratic plane stress elements was generated (Fig. 4d). The individual stiffness tensor was calculated and assigned to each set of elements representing one grain. The 


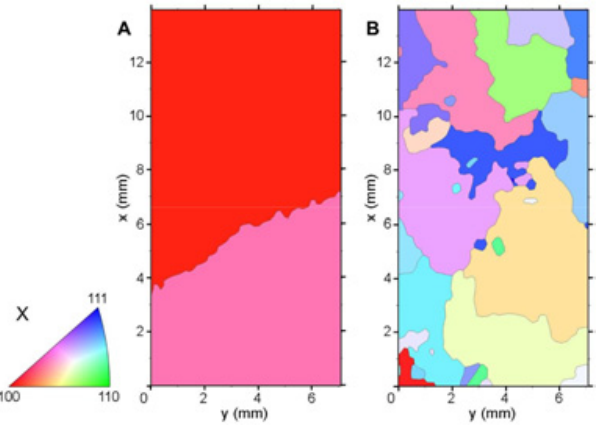

Figure 5. Grain orientation maps (X axis) of a bicrystalline (A) and a polycrystalline specimen (B).

boundary conditions for the FEM problem are illustrated in Fig. 4a. The areas adjacent to the specimen gauge section not characterized by EBSD were modeled as isotropic material. A constant displacement $d$ in $x$ direction was imposed. The stress and strain distribution were calculated using the CalculiX FEM solver [19].

\section{Results}

\subsection{Grain orientation}

In Fig. 5 results of the grain orientation mapping on a bicrystalline (A) and a polycrystalline specimen (B) are shown. The grain orientation is indicated by a color code referring to the orientation of the crystal $X$ axis in the standard triangle. Though necessary in order to fully characterize the grain orientation, the $Y$ and $Z$ axis are not represented here. As can be learned from the orientation maps, the bicrystalline specimen consists of two grains separated by an inclined high angle boundary. In the polycrystalline specimen 38 grains were detected, randomly oriented without any specific texture. The grain boundaries are arbitrarily shaped, as can be expected for a cast material. The grain size ranges between 1 and $2 \mathrm{~mm}$. In some regions small remains of truncated grains appear. Such grains were neglected for FE modeling.

\subsection{Mechanical anisotropy}

The local stiffness and Schmid factor maps corresponding to the grain orientation are shown in Fig. 6 (for this representation uniaxial loading in $x$ is assumed). In the bicrystalline specimen the grains are in serial configuration relative to the loading direction. Therefore, it can be expected that the stress distribution will be rather homogeneous. The strain, however, should concentrate to the upper grain, due to its lower stiffness. Considering the Schmid factors, one can deduce that in the lower grain, the maximum resolved shear stress will be increased. Thus, it can be expected that slip will be locally activated.

In the polycrystalline specimen the elastic modulus covers a broad range from 125 up to $300 \mathrm{GPa}$. Due to elastic incompatibility, it can be expected that an inhomogeneous elastic stress and strain distribution will arise in the microstructure. The stiffness is relatively low in the lower half of the specimen and high in the mid part. Therefore, strain concentrations are likely to occur in the

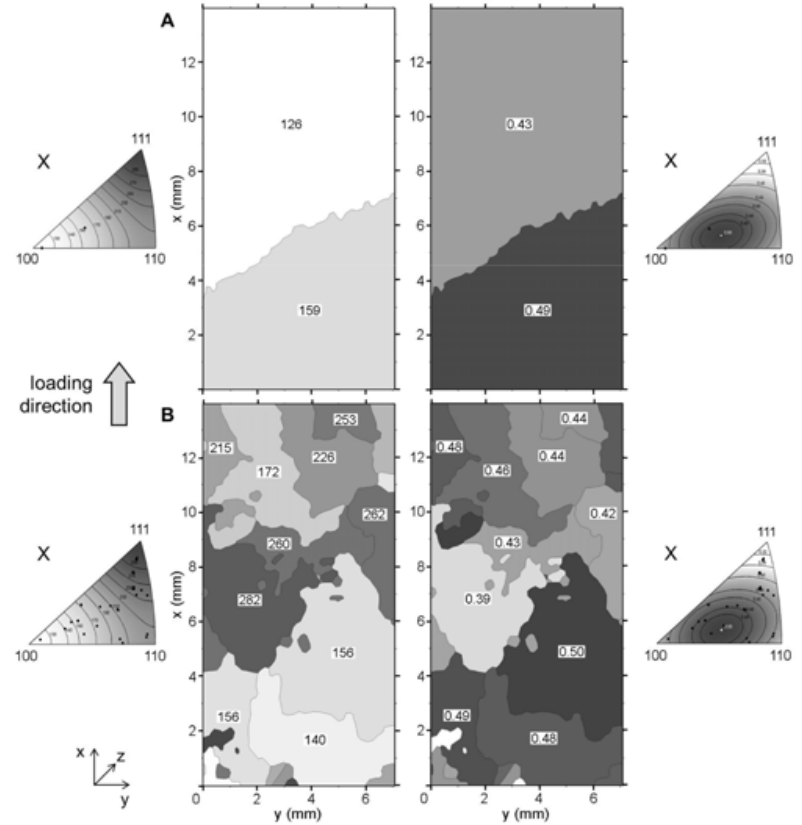

Figure 6. Local stiffness (left) and Schmid factor map (right) of specimens A (top) and B (bottom). Numbers represent stiffness and Schmid factor values.

lower part. A more detailed analysis of the elastic stress distribution and resolved shear stresses in specimen B can only be attained by FEM simulation.

\subsection{Elastic strain and stress distribution}

In Fig. 7 the elastic stress and strain distributions are shown for a total strain of $0.4 \%$ (A) and $0.3 \%$ (B). The results are in good agreement with the expectations described in the previous section. For specimen A, the von Mises stress in both grains is not fully homogeneous (as can be expected for a perfectly horizontal grain boundary), but differ by $100 \mathrm{MPa}$. The majority of the imposed strain is localized in the upper grain. The decomposition of the total strain follows precisely the portions of the two grains at the right side of the specimen, where the clip gauge was applied.

During the first loading cycle, the test was stopped at $0.4 \%$ and the local strain was measured by ARAMIS. As depicted on the right hand side of Fig. 7, the measured elastic strain is in good agreement with the simulation results regarding both the strain localization and its absolute values. Some strain concentrations at the left edge and the upper right corner are likely artefacts.

The von Mises stress distribution in specimen B reveals local stresses considerably higher than a macroscopic estimation would suggest. (Assuming an isotropic stiffness of $200 \mathrm{GPa}$, the stress resulting from an imposed strain of $0.3 \%$ would be $600 \mathrm{MPa}$ ). The calculated strain distribution essentially follows the local stiffness shown in Fig. 6. The measured local strains correspond well to the simulation in the lower part of the specimen. However, the strain localization in the upper right corner of specimen $B$ is not predicted by the model. 

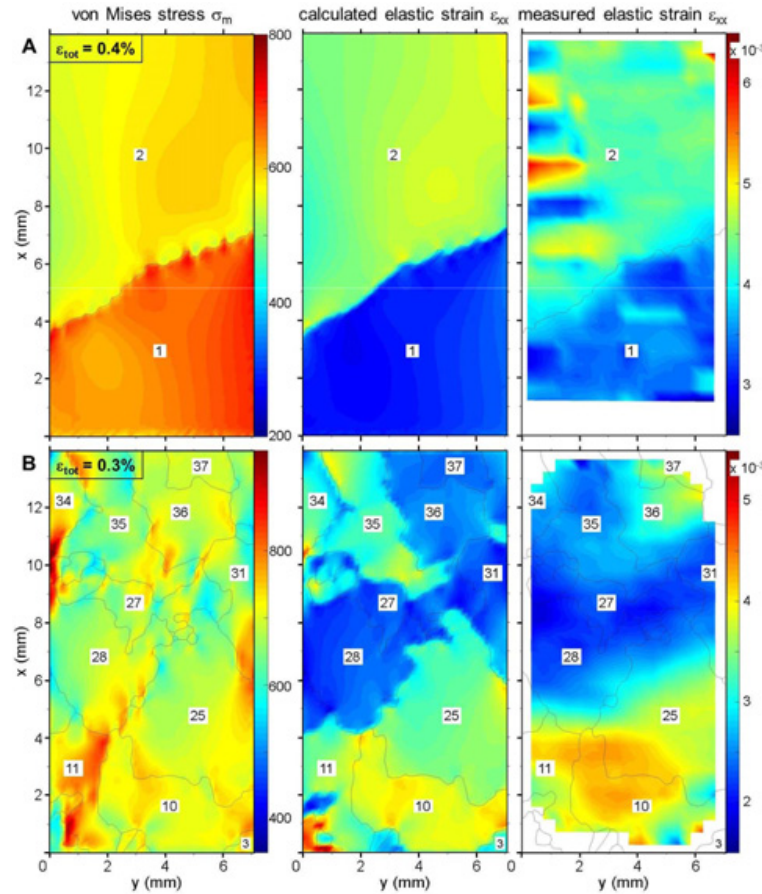

Figure 7. von Mises stress and strain $\left(\varepsilon_{\mathrm{xx}}\right)$ distribution in specimens A and B in comparison to ARAMIS measured local strain. Numbers represent grain indices.

\subsection{Onset of plasticity}

In order to identify the onset of plasticity in the first loading cycle, ARAMIS measurements were carried out after unloading the specimens subsequent to stepwise increases of the total strain. The results are presented in Fig. 8 along with the maximum resolved shear stress calculated based on the local stress tensor resulting from the FEM simulation. For specimen A, the amount of residual plastic deformation increases significantly at a macroscopic strain level around $0.65 \%$. The regions where plastic deformation is detected correspond well to the simulated shear stress concentrations. In specimen B the local onset of plasticity seems to occur at a macroscopic strain of $0.40 \%$. The strain measurements show that the local plasticity is in quite good consistence with the shear stress maxima in the lower and the upper left part of the specimen. However, the plastic deformation in the upper right corner is not predicted by the simulation.

Furthermore, knowing that plastic deformation occurs once the critical resolved shear stress $\tau_{\mathrm{c}}$ is reached, an estimation about this material-inherent constant can be deduced from the maximum shear stress values in Fig. 8, which range around $470 \mathrm{MPa}$ for both specimens. This is in rough accordance with values given by Pollock et al. [1], who proposed a critical resolved shear stress of $400 \mathrm{MPa}$ for MAR-M200 alloy. It should be noted that the $\tau_{\mathrm{c}}$ value estimated here is probably too high, since the onset of plasticity might occur already for lower stresses, producing micro-plastic deformations which were not detected at the specimen surface.

Additionally, local plastic deformation due to the activation of slip systems was identified via slip traces on the specimen surface. The fatigue test on specimen B was

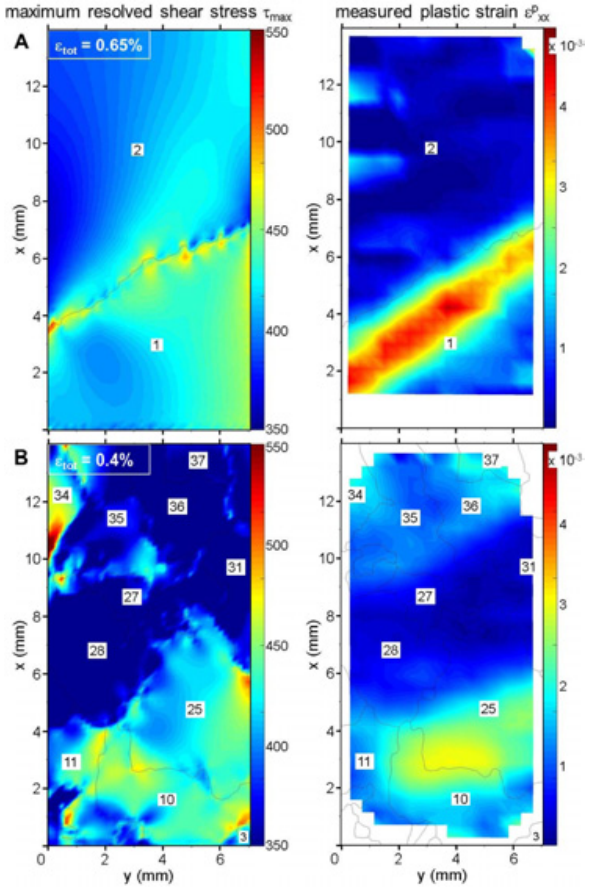

Figure 8. Maximum resolved shear stress in comparison to residual plasticity measured by ARAMIS after unloading specimens A and B in the first loading cycle.
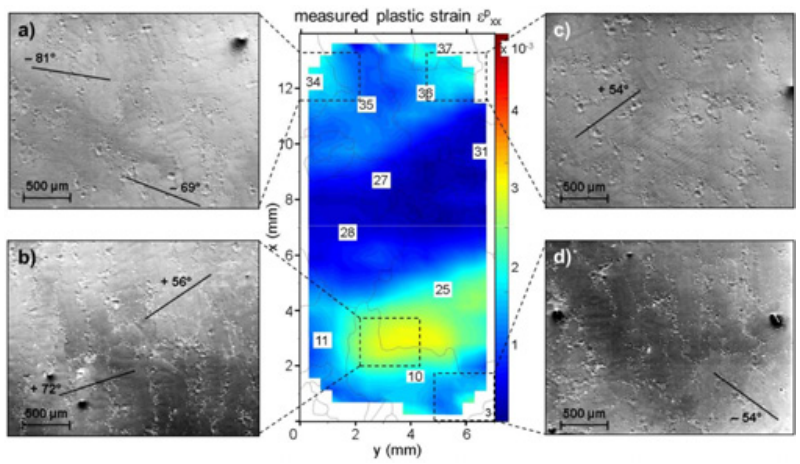

Figure 9. SEM images of specimen B showing slip traces due to local plastic deformation after 100 loading cycles.

interrupted after 100 cycles. SEM examination revealed slip traces in grains where local plastic deformation was detected (Fig. 9). As shown in Table 1, the measured slip mark angle corresponds well with calculated values. Thus, it can be concluded that the activation of slip systems was correctly predicted by the model.

\subsection{Crack initiation}

Specimen A was run until failure occurred at 39000 cycles. From Fig. 10 it can be seen that plastic deformation in the lower grain had substantially increased. SEM analysis revealed several cracks, which obviously had grown from slip traces in the lower grain. The measured angle to the loading direction $\left(-47^{\circ}\right)$ was very close to the predicted value $\left(-45^{\circ}\right)$. The largest crack, visible in subfigure a and $\mathrm{d}$, had grown over the complete lower grain and finally led to failure of the specimen. When the crack reached the grain boundary, its growth direction 
Table 1. Accordance of calculated active slip systems with measurements for a selected number of grains $i$ in specimen B.

\begin{tabular}{|c|c|c|c|c|c|c|c|}
\hline \multicolumn{4}{|c|}{ highest Schmid factor } & \multicolumn{4}{|c|}{ 2nd highest Schmid factor } \\
\hline$i$ & $s^{\max , \mathrm{i}}$ & $\begin{array}{c}\text { active slip } \\
\text { system }\end{array}$ & $\begin{array}{c}\theta^{\max , \mathrm{i}} \\
\text { calculated }\end{array}$ & $s^{\max 2, \mathrm{i}}$ & $\begin{array}{l}\text { active slip } \\
\text { system }\end{array}$ & $\begin{array}{c}\theta^{\max 2, \mathrm{i}} \\
\text { calculated }\end{array}$ & $\begin{array}{c}\theta \\
\text { measured }\end{array}$ \\
\hline 34 & 0.48 & $\{1-11\}\langle 011\rangle$ & $-81^{\circ}$ & 0.38 & $\{11-1\}\langle 1-10\rangle$ & $+72^{\circ}$ & $-81^{\circ}$ \\
\hline 35 & 0.46 & $\{111\}\langle 01-1\rangle$ & $-66^{\circ}$ & 0.43 & $\{1-11\}\langle-1-10\rangle$ & $+36^{\circ}$ & $-69^{\circ}$ \\
\hline 36 & 0.44 & $\{1-11\}\langle-1-10\rangle$ & $+\mathbf{5} 7^{\circ}$ & 0.41 & $\{11-1\}\langle 1-10\rangle$ & $-62^{\circ}$ & $+\mathbf{5 4}$ \\
\hline 25 & 0.50 & $\{1-11\}\langle-1-10\rangle$ & $+\mathbf{5 8}$ & 0.48 & $\{11-1\}\langle 1-10\rangle$ & $-52^{\circ}$ & $+56^{\circ}$ \\
\hline 10 & 0.48 & $\{11-1\}\langle 1-10\rangle$ & $-39^{\circ}$ & 0.46 & $\{1-11\}\langle-1-10\rangle$ & $+72^{\circ}$ & $+72^{\circ}$ \\
\hline 3 & 0.48 & $\{-111\}\langle-1-10\rangle$ & $-56^{\circ}$ & 0.38 & $\{11-1\}\langle 1-10\rangle$ & $+66^{\circ}$ & $-54^{\circ}$ \\
\hline
\end{tabular}
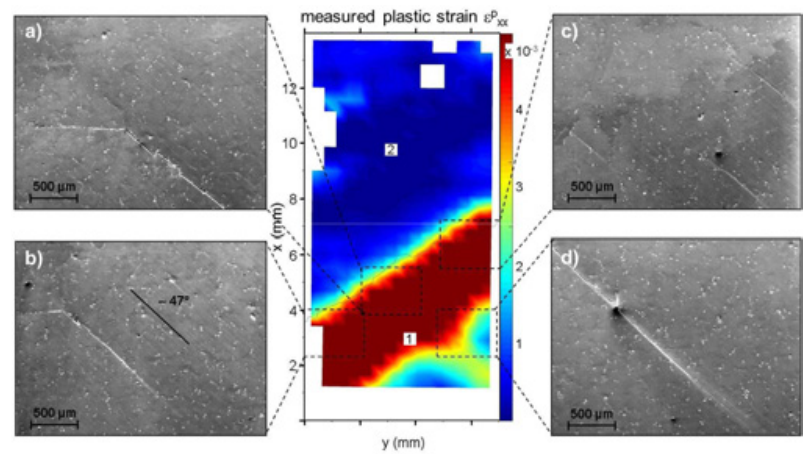

Figure 10. SEM images of specimen B after failure showing slip traces and cracks after 35000 loading cycles.

changed to a $90^{\circ}$ angle. The same behavior can be observed for the smaller crack in subfigure b. The upper grain was completely free of slip activity.

Furthermore, fatigue tests on a polycrystalline specimen carried out in the LC-SEM confirmed the mechanism of local slip band formation and crack initiation parallel to slip marks, leading to failure of the specimen (Fig. 11). After 1000 cycles local accumulation of slip traces was observed. The density and amplitude of slip traces intensified continuously, until at 10000 cycles the first stage I cracks appeared, which than joined to a larger crack leading to failure of the specimen. Thus, stage I crack initiation and crack propagation could be analyzed in-situ.

\section{Summary and Conclusions}

Based on the grain orientation mappings carried out in this study it was possible to characterize the anisotropic mechanical properties of bi- and polycrystalline specimens in terms of grain stiffness and Schmid factors. For precise analyses of the microscopic strain and stress distribution a micro-mechanical FE model was established. The simulated strain distribution corresponded well with the measured local strains. Furthermore, the experiments showed that calculated resolved shear stress maxima gave a good indication about the onset of plasticity in the first loading cycle of a fatigue test on both specimens.

However, some discrepancies between the simulation and the experimental findings remained. In this context, the assumptions taken for the micro-mechanical model, which possibly influence the results, should be discussed: Since the 3D grain morphology could not be measured,
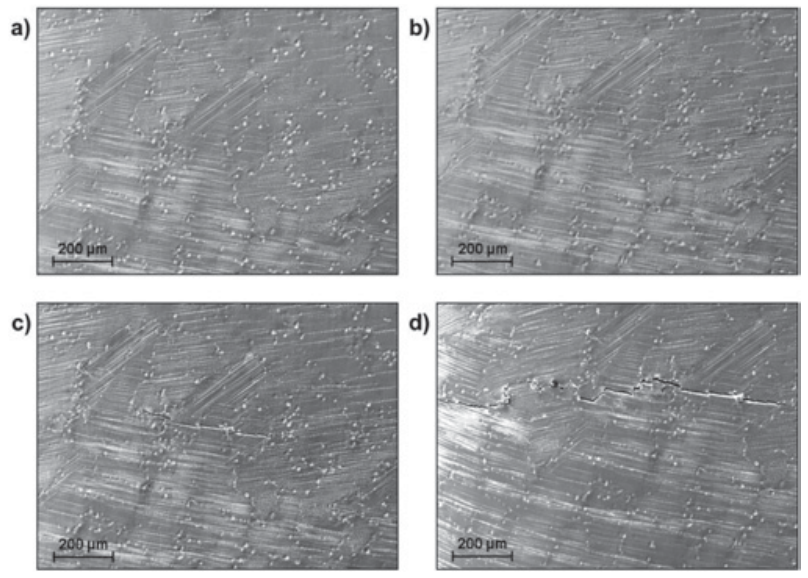

Figure 11. In-situ analysis of a polycrystalline specimen in the large chamber SEM under cyclic loading, showing slip traces and crack formation after a) 1000, b) 6000, c) 10000 and d) 11200 cycles.

the microstructure was modeled in 2D using plane stress elements. For this purpose, it was assumed that the grains were sufficiently large compared to the specimen thickness, so grain boundaries were oriented perpendicular to the specimen surface. However, Vehoff et al. [8] have shown that the inclination of grain boundaries in the third dimension substantially affects the stresses calculated by 3D FE models.

The role of micro-plastic deformation for stage I crack initiation was clearly demonstrated by the experimental studies in this work, including in-situ fatigue tests carried out in the LC-SEM. However, it must be pointed out that the FE model used in this work is only capable of predicting the onset of plastic deformation. For modelling of micro-plasticity, which is of high relevance for crack initiation, crystal plasticity routines are needed. Dunne et al. [17] proposed to analyze locally accumulated plastic strain under cyclic loading as an indicator for preferred crack formation, using a crystal plasticity FE model.

Thus, further experiments and improved simulations must be carried out in order to better illuminate the influence of mechanical anisotropy in complex microstructures for the formation of stage I cracks and their influence on fatigue life.

The authors thank C. Schulbert, Institute for Paleontology, Friedrich-Alexander-Universität Erlangen-Nürnberg, for the use of SEM and EBSD equipment. 


\section{References}

[1] T.M. Pollock, S. Tin, J. Prop. Power 22, 361-374 (2006)

[2] H.J. Christ, Ermüdungsverhalten metall. Werkstoffe (1998)

[3] K.J. Miller, M.F.E. Ibrahim, Fatigue Eng. Mater. Struct. 4, 263-277 (1981)

[4] A. Weidner, D. Amberger, F. Pyczak, B. Schönbauer, S. Stanzl-T., H. Mughrabi, Int. J. Fat. 32, 872-878 (2010)

[5] Y. Murakami, T. Nomoto, T. Ueda, Y. Murakami, Fatigue Fract. Eng. Mater. Struct. 23, 903-910 (2000)

[6] P. Neumann, H. Vehoff, H. Heitmann, BMFT-FB-T 84-040 (1984)

[7] T. Seifert, H. Riedel, Int. J. Fatigue 32, 1358-1367 (2010)

[8] H. Vehoff, A. Nykyforchyn, R. Metz, Mat. Sc. Eng. A387-389, 546-551 (2004)
[9] M. Oja, K.S. Ravi Chandran, R.G. Tryon, Int. J. Fatigue 32, 551-556 (2001)

[10] R. Nolte, PhD thesis, FAU Erlangen-Nürnberg (2004)

[11] C. Krechel, Tagungsband Werkstoffprüfung, 133$138(2013)$

[12] MATLAB ${ }^{\circledR}$ R2011a, The MathWorks ${ }^{\circledR}$, Inc.

[13] F. Bachmann, R. Hielscher, H. Schäben, Ultramicroscopy 111, 1720-1733 (2011)

[14] H. Bunge, Texture Analysis in Materials Science (1993)

[15] W. Hermann, Superalloys, TMS, 229-238 (1996)

[16] C. Blochwitz, J. Brechbühl, W. Tirschler, Mat. Sc. Eng. A210, 42-47 (1996)

[17] F.P.E. Dunne, A.J. Wilkinson, R. Allen, Int. J. Plasticity 23, 273-295 (2007)

[18] Mesh2D, free software by B. Kaan Karamete

[19] CalculiX, free software three-dimensional structural finite element program by G. Dhondt and K. Wittig 